\title{
New optical, acoustic, and electrical diagnostics for the developing world
}

S. Neale, C. Witte, Y. Bourquin, C. Kremer, A. Menachery, et al.

S. L. Neale, C. Witte, Y. Bourquin, C. Kremer, A. Menachery, Y. Zhang, R. Wilson, J. Reboud, J. M. Cooper, "New optical, acoustic, and electrical diagnostics for the developing world," Proc. SPIE 8251, Microfluidics, BioMEMS, and Medical Microsystems X, 825102 (3 February 2012); doi: 10.1117/12.924357 


\title{
New Optical, Acoustic, and Electrical Diagnostics for the Developing World
}

\author{
S.L. Neale, C. Witte, Y. Bourquin, C. Kremer, A. Menachery, Y. Zhang, R. Wilson, J. Reboud and \\ J.M. Cooper* \\ Biomedical Engineering Research Division, School of Engineering, University of Glasgow, Rankine \\ Building, Glasgow, G128LT, UK
}

\begin{abstract}
Infectious diseases cause 10 million deaths each year worldwide, accounting for $\sim 60 \%$ of all deaths of children aged 5 14. Although these deaths arise primarily through pneumonia, TB, malaria and HIV, there are also the so called "neglected diseases" such as sleeping sickness and bilharzia, which have a devastating impact on rural communities, in sub-Sahara Africa. There, the demands for a successful Developing World diagnostic are particularly rigorous, requiring low cost instrumentation with low power consumption (there is often no fixed power infrastructure). In many cases, the levels of infection within individuals are also sufficiently low that instruments must show extraordinary sensitivity, with measurements being made in blood or saliva. In this talk, a description of these demands will be given, together with a review of some of the solutions that have been developed, which include using acoustics, optics and electrotechnologies, and their combinations to manipulate the fluid samples. In one example, we show how to find a single trypanosome, as the causative agent of sleeping sickness.
\end{abstract}

Keywords: Optics, Acoustics, Dielectrophoresis, Diagnostics, Developing World

\section{INTRODUCTION}

There are many diseases prevalent predominantly in the Developing World and these can be put into two broad groups of major diseases which attract a large amount of attention from both health professionals and the academic community and other overlooked diseases which receive substantially less attention. However whether the disease is a major disease such as malaria or an overlooked disease such as human African trypanosomiasis (also known as sleeping sickness) the challenge of creating a diagnostic device suitable for the Developing World is the same.

Traditionally research in diagnostic technologies and devices has been carried out with a view to solve problems arising from the Developed World community. This has resulted in a wide range of systems that make use of well-funded laboratories within a highly regulated environment. However these systems are not adapted to the need of more than half of the world's population, living within the Developing World and resource-limited settings, while the needs for better diagnostics extend far beyond these areas.

More specifically, pathogens are showing increasing resistance to first line therapy antibiotics, while drug costs are rising and efficient diagnostics would help reduce the spreading of drug-resistant diseases. Furthermore, recent experiences have shown that local epidemics are more and more difficult to constrain and accessible diagnostics would be an efficient barrier against a spread to pandemics, due to the increasing interconnectivity of different populations.

Recently, in addition to intrinsic controls on the sample at the microscale, that have led to significant improvements in sensitivities and reproducibility, the advent of microfluidic technologies and the miniaturisation induced by microelectronics has led to the development of 'point-of-care' devices (POC), portable, self-sufficient devices, with the intent of bringing the diagnostics closer to the patient, in a faster timeframe ${ }^{1}$. In the Developed World, these have brought cost-effective and frequent diagnostics to better manage healthcare and in particular chronic conditions, such as glucose sensors for diabetes. However the benefits of deploying and adapting these technologies to the Developing World are probably even greater ${ }^{2}$.

*jon.cooper@glasgow.ac.uk; phone 44 1413304931;

http://www.gla.ac.uk/schools/engineering/research/divisions/biomedical/advancedmedicaldiagnosticsloac/

Microfluidics, BioMEMS, and Medical Microsystems X, edited by Holger Becker, Bonnie L. Gray, Proc. of SPIE Vol. 8251, 825102 - (C) 2012 SPIE · CCC code: 0277-786X/12/\$18 - doi: 10.1117/12.924357 
Taking into account the requirements of resource-poor settings, such as the lack of power, refrigeration, skilled staff or waste management, research has been focused on cost-effective and robust substrates such as plastics ${ }^{3}$ or even paper ${ }^{4}$.

These tend to use capillarity to drive fluid movements and thus do not require additional equipment. They also incorporate complex fluidic paths and enable some chemical reactions ${ }^{5}$. However their applications to diseases where high sensitivity is required, is not straightforward. In particular, diseases caused by parasites, such as malaria or sleeping sickness, both accounting for a high burden in Africa for example, require extreme sensitivities below 1000 parasites per millilitre of blood ${ }^{6}$, within 5 billion red blood cells. At these sensitivities, sample enrichment procedures are required to bring this ratio to a more manageable level. When performing blood smear microscopy, the conventional detection technique, enrichment is usually performed with a centrifuge, but this is rarely available on site, leading to poor sensitivity.

Here we show how new developments in the area of microfluidics open the way to cheap POC diagnostics for these diseases using acoustic and electromagnetic waves to perform on-chip sample processing and enrichment. More particularly, we show how the use of phononic structures can bring very efficient acoustic microfluidics, to a disposable and versatile platform, with low power requirements. This is completed by optically induced dielectrophoresis (Optoelectronic Tweezers, OET) to specifically separate particles or cells based on their electrical properties. Finally we show how they can be combined to offer a valid platform for POC diagnostics in the Developing World.

\section{ACOUSTICS}

It was Lord Rayleigh who in 1885 who mathematically showed the existence of non-dispersive wave propagation confined to the surface of an elastic solid ${ }^{7}$. The Rayleigh wave or surface acoustic wave (SAW) has a strong shear component normal to the direction of propagation and a longitudinal component in the direction of propagation. Although the majority of the wave's energy is confined to the surface an exponential decay of the displacements follows with depth. However, when propagating on a substrate with finite thickness it is clear that if the wavelength is long enough we can observe the surface wave on the other side. Such waves propagating in elastic plates were first studied by Lamb and they bear his name ${ }^{8}$. There are other kinds of surface acoustic waves but we will only discuss Rayleigh and Lamb waves and indeed treat them as if they were both equivalent and refer only to surface acoustic waves.

The interdigitated transducer was invented almost fifty years ago, the birth of modern SAW devices could be traced to the patents filed independently in 1963 by Rowen ${ }^{9}$ and Mortely ${ }^{10}$, which proposed the concept of metal gratings for planar surface acoustic wave devices. However it is curious to note that a similar tapped delay line structure was independently developed at the Academy of Aerospace Instrumentation in the then Soviet Leningrad in the early 60's but was not published or patented for security reasons. The demonstration of White and Voltmer in $1965^{11}$ of launching a surface acoustic wave on quartz using interleaved conducting combs showed that the time domain could be captured in millimetre space through the use of surface waves traveling at much slower acoustic velocities. This stimulated much basic research for the next few years with an exponential growth occurring in the early 1970's. SAW technology is now found extensively in consumer electronics and wireless telecommunications.

The surface acoustic waves generated on a polished piezoelectric substrate are usually those first described by Rayleigh. The generating structure consists of a series of interleaved metal lines with each alternate line is connected together making a one terminal pair driving point. Due to the fact that an electric field can cause a mechanical deformation in a piezo material we can drive the structure at a resonance frequency where the distance between alternate electrodes is the same as the wavelength of the surface wave then a high amplitude bidirectional surface wave radiates from the transducer.

The first use of surface acoustic waves in relation to microfluidics was described by Moroney ${ }^{12}$ in 1990 where Lamb waves were used in order to achieve a pumping action within a closed microfluidic channel and the use of Rayleigh waves to provide a pumping force for a droplet by Kurosawa ${ }^{13}$ in the mid 90's. The distinct advantage working with discrete droplets is low surface area to volume, meaning very small quantities of sample can be processed without losing too much of what you want to measure to the sides of a channel for instance.

A surface acoustic wave comprises of longitudinal and transversal components which propagate along the air/solid interface as the wave impinges on a liquid droplet, the surface acoustic wave becomes leaky, the transversal component is damped and in turn radiates (or leaks) a compressional wave into the liquid, this refracted wave refracts at the 
Rayleigh angle and is determined by the ratio of the speed of sound in the liquid over the speed of the Rayleigh or surface wave. There are two useful fluidic effects arising from this interaction, firstly the refracted wave having momentum can impart that momentum on the drop by exerting a radiation pressure causing deformation and ultimately movement when the resultant force exceeds that of the force of retention that the substrate exerts on the liquid, secondly the amplitude of the wave reduces as it passes under the drop due to damping and this gives rise to a second order effect called acoustic streaming within the drop and this is useful for sorting and mixing. Needless to say both effects are frequency dependent due to the fact that the acoustic absorbance of the liquid will be frequency dependent and hence the amount of damping will depend on the frequency used.

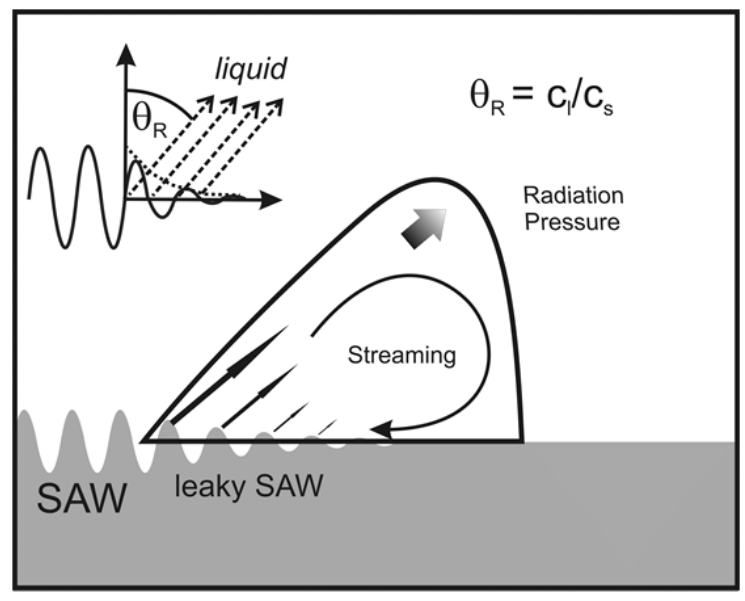

Figure1: Illustrating the two effects that an incoming surface acoustic wave can exert on a liquid droplet. The refracted compressional wave in the droplet has a momentum associated with it and this can be imparted to the drop on incidence with the air/liquid interface. The inset shows how the Rayleigh angle is defined and calculated as the ratio of the speed of sound in liquid over speed of sound in the solid. As the surface wave is damped by the liquid a second order flow develops in the droplet leading to what is called acoustic streaming. As the frequency is increased the absorption of the wave's energy becomes more efficient leading to great streaming and less actuation.

Using SAW microfluidic manipulations, we demonstrate here a bead-based immunoassay for the diagnosis of an infectious disease. The immunoassay is based on the manipulation and imaging of beads in a microlitre droplet on a planar surface for the detection of interferon- $\gamma$, a biomarker used in the diagnosis of tuberculosis. The control of particles using SAW allows the full integration of all the immunoassay process steps.

The device comprised a tapered interdigitated transducer (IDT), fabricated using standard photolithography and deposition of titanium and gold on a lithium niobate wafer. In this particular type of IDT, the position of the generated waves is tunable by the input frequency giving a way to precisely control the streaming inside the droplet and manipulate beads $^{14}$. This is an alternative method of controlling the acoustic fields to the previously demonstrated method of utilizing phononic crystals ${ }^{15,16}$. The acoustic waves were coupled into a disposable chip as Lamb waves through a thin layer of a water-based gel (KY jelly - Johnson \& Johnson). Only a small part of the chip was in contact with the substrate, the free space under the cover slip allowing positioning in a fluorescent microscope as shown in Figure 2. 


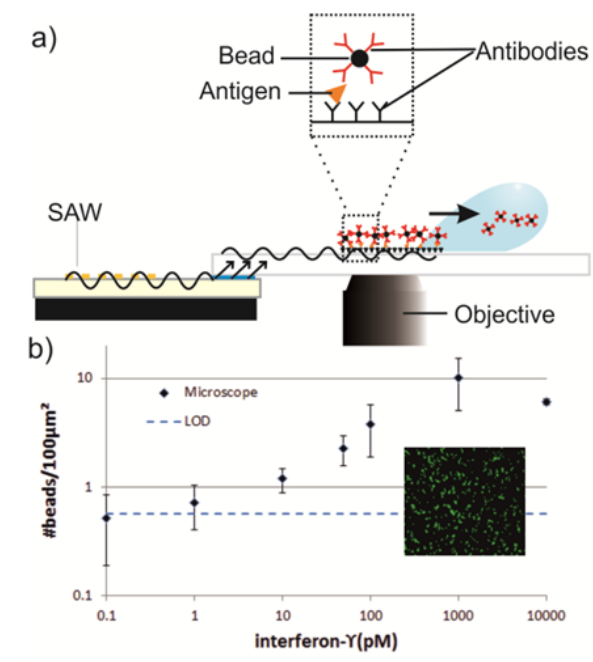

Figure 2: a) The SAW are generated by the electrodes and propagate on the piezoelectric surface; they are coupled into the chip through a thin layer of water-based gel and refracted into the droplet resulting in fluid streaming. The beads bound to the surface via the antibody-antigen-antibody link are optically detected. b) Dose-response curve of the immunoassay for interferon- $\gamma$. Results show the mean of 3 repetitions and error bars are the standard deviation. The inset shows a fluorescent image of the detected beads on the surface after the washing step.

Monoclonal antibodies were covalently bound onto a defined $3 \mathrm{~mm}$ spot on the disposable chip. Latex beads (Figure $2 \mathrm{~b}$, $2 \mu \mathrm{m}$, fluorescent, carboxylate) were functionalised with a second monoclonal antibody (Figure 2a).

All of the steps of the designed sandwich immunoassay were controlled by the ability of the SAW to manipulate the fluid and particles within it $^{17}$. A drop containing the analyte, human interferon- $\gamma$ (Sigma Aldrich, I3265), was deposited on the spot of antibodies on the surface of the biochip. Using SAW streaming (frequency f1 $=13.2 \mathrm{MHz}, 320 \mathrm{~mW}, 30 \mathrm{~s}$ ), the beads were rapidly mixed increasing the speed of capture of the analyte. An angular momentum was then created in the droplet ( $\mathrm{f} 2=12.12 \mathrm{MHz}, 320 \mathrm{~mW}, 570 \mathrm{~s}$ ), to collect the beads near the surface covered by the second antibody, enhancing the binding kinetics. Finally, a third frequency excited at a higher power ( $\mathrm{f} 3=13.4 \mathrm{MHz}, 500 \mathrm{~mW}, 10 \mathrm{~s}$ ) enabled to wash away the drop with the unbound beads. The remaining beads, specifically attached to the surface, were then observed using a fluorescent microscope. Single beads were easily observed as shown in Figure 2b (inset) and counted using a public domain software (ImageJ).

A dose-response curve for interferon- $\gamma$, showing the number of beads per surface area as a function of the concentration of analyte, is presented in Figure $2 \mathrm{~b}$. The assay was performed in only $10 \mathrm{~min}$. The input power of the SAW actuation during the washing step was optimised to reduce non-specific binding, without compromising the specific binding. The limit of detection, determined as three times the standard deviation of the background signal, was situated under $10 \mathrm{pM}$, which is the limit required for the diagnostic of tuberculosis in the interferon- $\gamma$ release assay. The measurement dynamic range spanned three orders of magnitude (1-1000 pM), comparing favourably with other beads based techniques.

This technique showed a high sensitivity with good reproducibility on a disposable chip, opening the way to the diagnosis of tuberculosis as well as other infectious diseases. Furthermore, by using other mobile phone components, such as a CMOS sensor and a LED, it would be possible to replace the detection system with a low cost and smaller size device adapted for diagnostics in Developing Countries ${ }^{18}$. 


\section{DIELECTROPHORESIS}

The term dielectrophoresis (DEP) in its general form is defined as the effect where a particle undergoes movement as a result of the interaction of the induced dipole moment with a spatially varying electric field magnitude and phase ${ }^{19}$. The time averaged force is given by;

$$
F_{D E P}=\frac{1}{4} \varepsilon_{m} \operatorname{VRe}[C M] \nabla|E|^{2}+\frac{1}{2} \varepsilon_{m} \operatorname{VIm}[C M] \sum E^{2} \nabla \varphi
$$

where $\mathrm{V}$ is the volume of the particle and $\varepsilon_{\mathrm{m}}$ is the permittivity of the suspending medium. The first force term on the right hand side of the equation is responsible for directing particles towards or away from strong field regions and is referred to as conventional DEP, it is proportional to the in phase component of the dipole moment $(\propto \operatorname{Re}[\mathrm{CM}])$ and the field magnitude non-uniformity $\left(\nabla|\mathrm{E}|^{\wedge} 2\right)$. The second force term has to be considered only when particles are subject to a travelling electric field, resulting in an interaction between the out of phase component of the dipole moment $(\propto \operatorname{Im}[\mathrm{CM}])$ and the field phase non-uniformity term $\left(\sum \mathrm{E}^{\wedge} 2 \nabla \varphi\right)$.

The frequency response of a biological cell in a non-uniform electric field is normally characterized by a cross-over frequency $\left(f_{c r}\right)$, corresponding to a frequency where a cell experiences a transition from a negative (repulsive force) to a positive (attractive force) value, shown in Figure 3a). Using the same suspending medium conditions, cellular DEP responses close to the cross-over frequency are commonly used for biological separation ${ }^{20-22}$, it is found to mostly depend on the size, shape, membrane morphology and membrane integrity of the cell. Gascoyne et al. applied this label free cell discrimination technique to the area of medical diagnostics for the isolation of malaria-infected cells from blood $^{23}$.

a

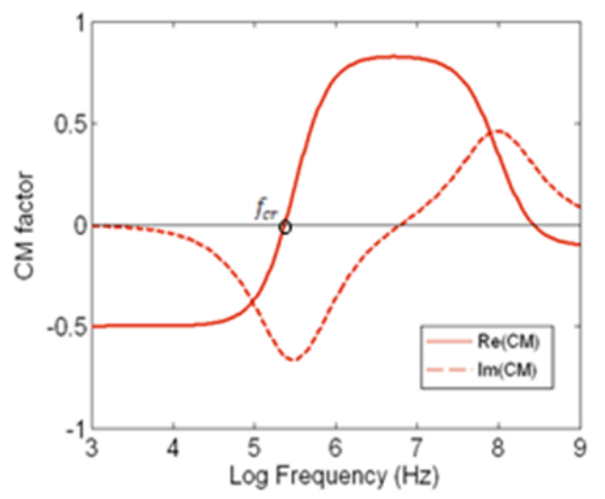

Figure 3 a) Modeled DEP response of a viable mammalian cell showing a transition from negative to positive regime around the cross-over frequency $\left(\mathrm{f}_{\mathrm{cr}}\right)$.

A study using DEP as a method of separating a range of different blood borne from infected mouse blood has been carried out. Firstly, experimental conditions required to induce differential positive and negative DEP movement of the parasites and RBCs was determined by measuring the crossover frequency at which the cells exhibit zero DEP motion. RBCs were pushed into the centre whereas the parasites were trapped at the electrode edges. Separation was achieved due to differences in frequency dependant polarisability between the red blood cells and the parasites. The separation of the parasites from the RBCs will make their detection and therefore the diagnosis of this disease possible despite the large numbers of RBCs to each parasite. 


\section{OPTOELECTRONIC TWEEZERS}

Optoelectronic Tweezers (OET) is a method of optically inducing dielectrophoresis by the selective illumination of a photoconductive layer. The light pattern creates a similar pattern of more conductive semiconductor as the light is absorbed creating charge carrier pairs which increase its conductivity. When a device is constructed so that an electric potential is placed across a liquid layer and the photoconductor the effect is to concentrate the applied electric field in the liquid just above the illuminated photoconductor. This was first demonstrated by Chiou et al. ${ }^{24}$ which demonstrated the effectiveness of this technique for manipulating many particles simultaneously. This is because this technique requires much less light to move particles than a conventional optical tweezer, indeed it has been shown that to create a trap with a similar stiffness you would need 470 times more light ${ }^{25}$. It has been shown that the optimum size light pattern to move a cell with is a light spot of similar size to the cell and the potential energy well for such a trapped cell has been simulated $^{26}$. As shown in Section 3 the DEP force is different for different species of cell with parasites having a different frequency response than RBCs. In this way OET has been used to separate live cells from dead cells ${ }^{24}$, viable but non motile sperm cells sperm cells from dead sperm cells ${ }^{27}$, embryo of different stage of development ${ }^{28}$ and trypanosomes from $\mathrm{RBCs}^{29}$.

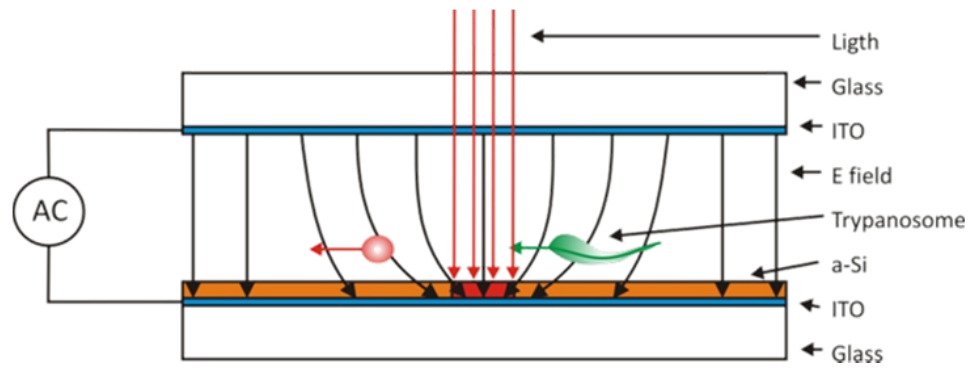

Figure 4, schematic diagram of the OET device with the electrical field lines being concentrated above the illuminated region of the a-Si photoconductor and the trypanosome ${ }^{29}$ are being attracted to the illuminated high field region and the erythrocytes (RBCs) are repelled.

Figure 4 shows how an OET device can be used to separate such parasites from RBCs to aid their detection for the diagnosis of the disease. It can be seen that the long thin parasites are attracted to one area of the device whilst the RBC are repelled providing a powerful sorting technique which is more useful than if both cell types felt a positive force but one was weaker than the other. The advantage of OET here is that the electrical pattern can be moved so that if there is an area where it is suspected that a parasite is present (which can often happen as the clinician will see something moving but not be able to positively identify it) a light pattern can be placed here repelling all the RBC but not the parasite aiding detection.

\section{COMBINATION OF ELECTRICAL AND ACOUSTIC FORCES FOR PARTICLE HANDLING}

Flexible detection instrumentations require automated and programmable sample preparation functions. It becomes very important when heterogeneous biological mixtures (e.g. blood) are used where it is necessary to isolate a certain type of cell (e.g. infected cell) for immediate diagnostic purposes or follow-up processes (e.g. cell lysis). The direct detection of an analyte within a complexly composed liquid is often unachievable. Subsequently, sample preparations steps which include cell or particle manipulation strategies like separation, sorting, trapping and concentration need to be integrated into a diagnostic system.

Non-intrusive dielectrophoretic ${ }^{19,30}$ and acoustophoretic ${ }^{31,32}$ approaches are intensively studied and show promising tools capable to provide the mentioned functions. Despite clear advantages of each method and the relative simple fabrication process, only a few studies are undertaken to combine both methods ${ }^{33,34}$. However, it would be favourable to be able to utilize electrical and mechanical forces for addressing several properties of cells and particles in complex mixtures. 
Considering dielectric properties, the size and the frequency dependent permittivity of cells in a certain medium can be used to characterize, differentiate, and hence separate different cell types. Occasionally, a clear difference in the electrical response occurs when there are opposing signs of the underlying DEP force. The generation of nonhomogenous electric fields is realised by gold electrodes on solid substrates, commonly. However, more dynamic and programmable DEP configurations are gained when using light induced dielectrophoresis ${ }^{23,35}$. Different light patterned electrode shapes are easily applied and reconfigurable when it is needed to design certain functions like focusing or trapping (Fig.5). Furthermore, light induced dielectrophoresis is truly able to tweeze single cells while controlling their position manually ${ }^{36}$.

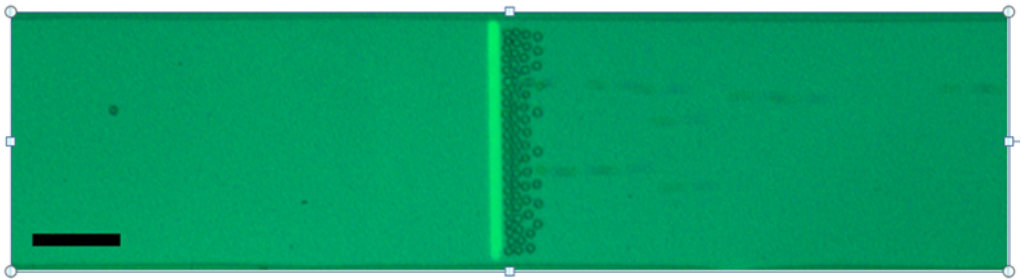

Fig. 5 Trapping of $6 \mu \mathrm{m}$ particles, liquid flow from the left $(0.5 \mu \mathrm{l} / \mathrm{min}, 15 \mathrm{Vpp}, 100 \mathrm{kHz}, 5 \mathrm{mS} / \mathrm{m}$, scale bar: $50 \mu \mathrm{m})$.

Microfluidic resonator structures for acoustophoretic manipulations consist of high acoustic impedance materials (e.g. Glass, silicon). The exciting of an acoustic wave is obtained with the actuation of a piezoelectric transducer. The propagating wave can be coupled to a resonator structure, filled with a cell or particle suspension. Here, an efficient and predictable applications of the acoustic radiation force is achieved when the resonator is frequency matched. In this case, an ultrasonic standing pressure wave can be generated in a microfluidic channel. Particles are forced to move to the pressure nodes or antinodes depending on their compressibility and density in relation to the solution (Fig.6). It has to be noted, that the acoustophoretic force can be applied along the whole channel length, while the DEP force localized to the electrode which creates the short-range electric field. A stable focused stream of particles can be achieved which leads to an enrichment along the pressure node (Fig.6c).

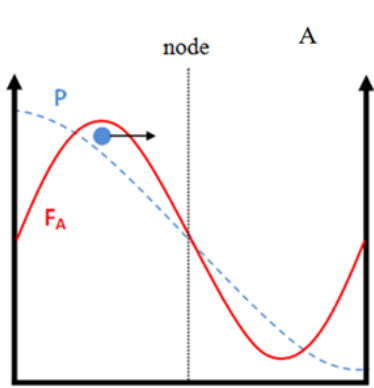

Channel width
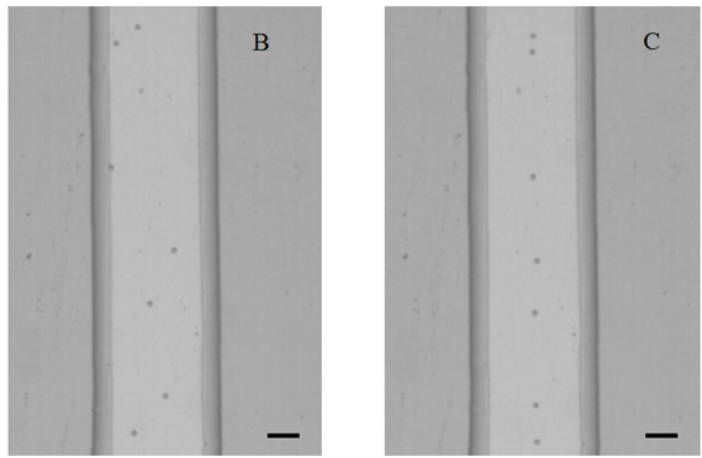

Fig.6: a) Pressure distribution $P$ and acoustic force $F_{A}$ acting on a bead. b) $200 \mu \mathrm{m}$ channel filled with $10 \mu \mathrm{m}$ polystyrene beads without fluid flow. Beads are randomly distributed in the channel. c) Acoustic actuation at $3.704 \mathrm{MHz}$ with $10 \mathrm{Vpp}$ leads to standing pressure wave where beads are focused into the pressure node (first harmonic) in the channel center (scale bar: 50um).

The fabrication of glass or silicon microchannels with integrated gold electrodes is realised by conventional photolithography, deposition, etching and bonding processes. The piezoelectric transducer is coupled to the microchannel and driven according to the resonance frequency of the whole device. DEP force and acoustic radiation force can be easily controlled by varying the applied voltage. In flow through applications the regulation of the hydrodynamic force is taken into account. 
For instance, a microchannel containing electrodes for DEP manipulations is holding particles against the flow (Fig.7 a). A negative DEP force causes the particle to stop and induces a lining up at the electrode front. Adding an acoustic force brings particle to the pressure nodes of the standing pressure wave inside the channel (Fig.7 b). The result is a dense packed aggregate of particles, precisely localized at a pressure node in a continuous flow. Here, the different forces acting on the particle (Fig.7 c) influence the shape of the aggregate. Furthermore, several aggregates along the electrode front can be merged switching from a multi-pressure node to a single node (first harmonic) operation. If a desired concentration is achieved, DEP force is switched off and further preparation steps downstream can be applied.
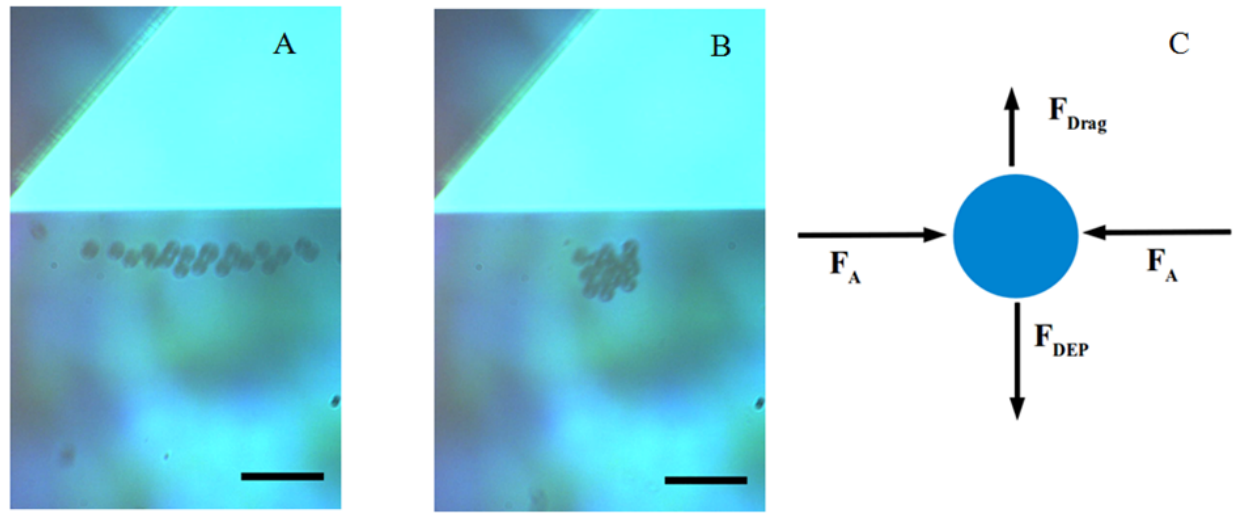

Fig.7: (a) 10um beads undergoing negative DEP force in a micro channel containing parallel electrodes. Beads are trapped against the flow and accumulation in form of lined up shape (1MHz, 10Vpp). b) Acoustic force is applied and beads move to the pressure node. A dense packed cluster is formed. c) Schematic representation of the forces acting on the bead (Scale bar $50 \mu \mathrm{m})$.

As the acoustophoretic and DEP force scale with radius of the particle, size dependent exclusion processes are easily implemented in the system. The applied potential for DEP manipulation can be reduced to a cut-off value which triggers the trapping of defined particle sizes and results in sample separations. The same effect but for acoustophoretic manipulation can be obtained when the amplitude of the acoustic pressure wave is regulated and coordinated with the drag force. The time to focus particles in the flow depends on the radius. Hence, smaller particles pass the electrode while other particles get trapped or deflected into collection zone.

In future, a sequential operation of acoustophoretic and dielectrophoretic based separation schemes will be applied. Differences in the acoustic contrast factor as well as differences in the polarizability of particles can be used to enhance preparation processes by means of $2 \mathrm{D}$ fractionation. This will lead to potential application in the field of diagnostics where rare cells types in biological samples need to be identified to investigate the health status of a patient.

\section{CONCLUSION}

There are many challenges to creating diagnostics devices for the Developing World as constraints such as low cost and the absence of the infrastructure of the Developed World make many of our conventional approaches unfeasible. Microfluidic approaches may find uses in Developed World diagnostics by producing information quicker or at the point of care instead of relying on sample being sent to a lab, however there may be much more to gain by using these techniques in the Developing World where there are fewer centralized facilities. In this paper we have described two promising technologies, acoustic and dielectrophoretic particle manipulation, that each could find applications in disease 
diagnostics through micro particle tweezing however we have also shown how they can be combined to produce an even more powerful micromanipulation technology.

\section{REFERENCES}

[1] Giljohann, D.A., Mirkin, C.A., "Review Article Drivers of biodiagnostic development” Nature 462, $461-464$ (2009)

[2] Ligler, F.S., “A Perspective on Optical Biosensors and Integrated Sensor Systems” Annu. Rev. Biomed. Eng. . 10:107-44 (2008).

[3] Stevens, D.Y., Petri, C.R., Osborn, J.L., Spicar-Mihalic, P., McKenzie, K.G., Yager, P., "Enabling a microfluidic immunoassay for the developing world by integration of on-card dry reagent storage" Lab Chip, 8, 2038-2045 (2008).

[4] Martinez, A.W., Phillips, S.T., Whitesides, G.M., "Three-dimensional microfluidic devices fabricated in layered paper and tape" PNAS vol. 105 no. 50 19606-19611 (2008).

[5] Nie, Z., Deiss, F., Liu, X., Akbulut, O., Whitesides, G.M., "Integration of paper-based microfluidic devices with commercial electrochemical readers" Lab Chip 10(22): 3163-3169 (2010).

[6] Wastling, S.L., Welburn, S.C., "Diagnosis of human sleeping sickness: sense and sensitivity" Trends in Parasitology, 27, 9, 394-402, (2011).

[7] Lord Rayleigh, "On waves propagating along the plane surface of an elastic solid". Proc. London Math. Soc. S1-17 (1): 4-11 (1885).

[8] Lamb, H., “On waves in an elastic plate”. Proc. Royal Soc. (London) Ser. A 93, 114 (1917).

[9] Rowen, J.H. (1963) U.S. Patent 3,289,114.

[10] Mortley, W.S. (1963) British patent 988,102

[11] White, R. M., and Voltmer, F. W., "Direct piezoelectric coupling to surface elastic waves". Appl. Phys. Lett. 7, 314-316 (1965).

[12] Moroney, R.M., White, R.M. and Howe, R.T. "Fluid motion produced by ultrasonic Lamb waves.” Proceedings of the IEEE 1990 Ultrasonics Symposium, 355-358 (1990).

[13] Kurosawa, M., Watanabe, T. and Higuchi, T. "Surface acoustic wave atomizer with pumping effect", Proceedings of IEEE Micro Electro Mechanical Systems MEMS '95, p25 (1995).

[14] Bourquin, Y., Reboud, J., Wilson R. and Cooper, J.M., “Tuneable surface acoustic waves for fluid and particle manipulations on disposable chips", Lab Chip, 10, 1898-1901 (2010).

[15] Wilson, R., Reboud, J., Bourquin, Y., Neale, S.L., Zhang, Y., Cooper, J.M., Lab Chip, 11, 323-328 (2011).

[16] Bourquin, Y., Wilson, R., Zhang, Y., Reboud, J. and Cooper, J.M., "Phononic Crystals for Shaping Fluids", Adv. Mat., 23, 1458-1462 (2011).

[17] Bourquin, Y., Reboud, R., Wilson, R., Zhang, Y. and Cooper, J.M., "Integrated immunoassay using tuneable surface acoustic waves and lensfree detection", Lab Chip, 11, 2725-2730 (2011).

[18] Bourquin, Y., Reboud, J., Wilson, R., Zhang, Y. and Cooper, J.M., "Low cost diagnostic device using mobile phone technologies", PGBIOMED 2 (2011).

[19] Pethig, R., "Dielectrophoresis: Status of the theory, technology, and applications", Biomicrofluidics, 4, pp. 022811-1 - 022811-35 (2010).

[20] Kim, U., Shu, C.W., Dane, K.Y., Daugherty, P.S., Wang, J.Y.J. and Soh, H.T. , "Selection of mammalian cells based on their cell-cycle phase using dielectrophoresis", P.N.A.S., 04, pp. 20708-20712 (2007). 
[21] Labeed, F.H., Coley, H. M., Thomas, H. and Hughes, M.P., “Assessment of Multidrug Resistance Reversal Using Dielectrophoresis and Flow Cytometry”, Biophysical Journal, 85 pp. 2028-2034 (2003).

[22] Vykoukal, J., Vykoukal, D.M., Freyberg, S., Alt, E.U. and Gascoyne, P.R.C., "Enrichment of putative stem cells from adipose tissue using dielectrophoretic field-flow fractionation", Lab Chip, 8, pp. 1386-1393 (2008).

[23] Gascoyne, P., Mahidol, C., Ruchirawat, M., Satayavivad, J., Watcharasit, P. and Becker, F. F. "Microsample preparation by dielectrophoresis: isolation of malaria", Lab Chip, 2, pp. 70-75 (2002).

[24] Chiou, P. Y., Ohta, A.T. and Wu, M.C., "Massively parallel manipulation of single cells and microparticles using optical images" Nature, 436, 370-372 (2005).

[25] Neale, S.L., Mazilu, M., Wilson, J.I.B., Dholakia, K. and Krauss, T.F., "The resolution of optical traps created by Light Induced Dielectrophoresis (LIDEP).” Optics Express, Vol. 15, No. 20, 12619-26 (2007).

[26] Neale, S.L., Ohta, A.T., Hsu, H.Y., Valley, J.K., Jamshidi, A. and Wu, M.C., “Trap profiles of projector based optoelectronic tweezers (OET) with HeLa cells", Optics Express 17, 5231-5239 (2009).

[27] Neale, S.L., Ohta, A.T., Hsu, H.Y., Valley, J.K., Jamshidi, A. and Wu, M.C., "Motile and non-motile sperm diagnostic manipulation using optoelectronic tweezers", Lab-on-a-chip, 10, 3213-3217 (2010).

[28] Valley, J.K., Garcia, M., Swinton, P., Neale, S., Hsu, H.Y., Jamshidi, A. and Wu, M.C., “Optoelectronic tweezers for quantitative assessment of embryo development stage", Micro Electro Mechanical Systems (MEMS), 2010 IEEE 23rd International Conference on, 943 - 946 (2010).

[29] Kremer, C., Neale, S., Barrett, M. Cooper, J., "Manipulating blood borne parasites with optoelectronic tweezers (OET)", IOP Photon10 (2010).

[30] Khoshmanesh, K., Nahavandi, S., Baratchi, S., Mitchell, A. and Kalantar-Zadeh, K., "Dielectrophoretic platforms for bio-microfluidic systems" Biosens Bioelectron, 26, 1800-1814 (2011).

[31] Laurell, T., Petersson F., and Nilsson, A., "Chip integrated strategies for acoustic separation and manipulation of cells and particles" Chem Soc Rev, 36, 492-506 (2007).

[32] Wang, Z. Zhe, J., "Recent advances in particle and droplet manipulation for lab-on-a-chip devices based on surface acoustic waves”, Lab Chip, 11, 1280-1285 (2011).

[33] Ravula, S.K., Branch, D.W., James, C.D., Townsend, R.J., Hill, M., Kaduchak, G., Ward, M. and Brener, I., “A microfluidic system combining acoustic and dielectrophoretic particle preconcentration and focusing" Sensors and Actuators B: Chemical, 130, 645-652 (2008).

[34] Wiklund, M., Gunther, C., Lemor, R., Jager, M., Fuhr, G. and Hertz, H.M., “Ultrasonic standing wave manipulation technology integrated into a dielectrophoretic chip" Lab Chip, 6, 1537-1544 (2006).

[35]Zarowna-Dabrowska, A., Neale, S.L., Massoubre, D., McKendry, J., Rae, B.R., Henderson, R.K., Rose, M.J., Yin, H., Cooper, J.M., Gu, E. and Dawson, M.D., "Miniaturized optoelectronic tweezers controlled by GaN micro-pixel light emitting diode arrays" Opt. Express, 19, 2720-2728 (2011).

[36] Valley, J.K., Ohta, A.T., Hsu, H.Y., Neale, S.L., Jamshidi, A. and Wu M.C., "Optoelectronic Tweezers as a tool for parallel single-cell manipulation and stimulation”, IEEE Transactions on Biomedical Circuits and Systems, Vol 3, 6, 424-431 (2009). 